\title{
Correlation Analysis between Mean Corpuscular Hemoglobin and Mean Corpuscular Volume for Thalassemia Screening in Large Population
}

\author{
Xiaoli Long1, Junyu Fang1,2, Lijun Yao', Qingqing Xiao¹, Tao Pan ${ }^{*}$ \\ ${ }^{1}$ Key Laboratory of Optoelectronic Information and Sensing Technologies of Guangdong Higher Educational \\ Institutes, Jinan University, Guangzhou, China \\ ${ }^{2}$ Family Planning Service Center of Dongguan, Dongguan, China \\ Email: ${ }^{*}$ tpan@inu.edu.cn
}

Received 26 July 2014; revised 11 September 2014; accepted 26 September 2014

Copyright (C) 2014 by authors and Scientific Research Publishing Inc.

This work is licensed under the Creative Commons Attribution International License (CC BY).

http://creativecommons.org/licenses/by/4.0/

(c) (i) Open Access

\begin{abstract}
Correlation analysis between the hematological parameters mean corpuscular hemoglobin (MCH) and mean corpuscular volume (MCV) for thalassemia screening in large population was discussed. A total of 4920 peripheral blood samples of reproductive age persons were collected from Guangdong province of China. The hematological parameters MCH and MCV values of samples were first measured, and then the DNA analyses for thalassemia were conducted. All samples were composed by 4463 non-thalassemia and 457 thalassemia, and among 457 thalassemia samples, 311 were $\alpha$-thalassemia, 133 were $\beta$-thalassemia, and 13 were $\alpha \& \beta$-thalassemia. In accordance with non-thalassemia, thalassemia, $\alpha$-thalassemia, $\beta$-thalassemia, $\alpha \& \beta$-thalassemia and the entire group itself, a total of six sample groups were divided. The corresponding correlation coefficients between the measured MCH and MCV values for the six sample groups were $0.880,0.968,0.966$, $0.962,0.980$ and 0.965 respectively. For the thalassemia carriers, highly significant correlation between MCH and MCV were observed. The fitting equations between MCH and MCV values were also obtained. The results indicated that the feasibility for thalassemia screening using MCV or MCH independently as parameter, and provided suitable strategy to select parameters and models for thalassemia screening in large population.
\end{abstract}

\section{Keywords}

Thalassemia Screening, Large Population, Mean Corpuscular Hemoglobin, Mean Corpuscular

*Corresponding author.

How to cite this paper: Long, X.L., Fang, J.Y., Yao, L.J., Xiao, Q.Q. and Pan, T. (2014) Correlation Analysis between Mean Corpuscular Hemoglobin and Mean Corpuscular Volume for Thalassemia Screening in Large Population. American Journal of Analytical Chemistry, 5, 901-907. http://dx.doi.org/10.4236/ajac.2014.514098 


\section{Volume, Correlation Analysis}

\section{Introduction}

Thalassemia is a group of common monogenic disorders of hemoglobin synthesis, and it affects individuals from many parts of the world, including Southern China, where it has a high prevalence and incidence and has caused serious health damage [1]-[3]. In Southern China, the rates of gene carriers in the population of Guangxi and Guangdong provinces are as high as $24.50 \%$ and $11.07 \%$, respectively [2] [3]. This serious disorder is caused by partial or total mutations that reduce or abolish the synthesis of $\alpha$ - or $\beta$-globin chains of the hemoglobin molecules, which will result in hemolytic anemia. At present, the disease cannot be cured, except through hematopoietic stem cell transplantation [4]. The most fundamental prevention methods include premarital and prenatal thalassemia screening in a large population. According to insufficient number of globin chains's type, thalassemia mainly includes three categories: $\alpha$-globin chain relative reduction is called $\alpha$-thalassemia, $\beta$-globin chain relative reduction is called $\beta$-thalassemia, and both $\alpha$ - and $\beta$-globin chain relative reduction is called coinheritance of $\alpha$-thalassemia and $\beta$-thalassemia (was simply denoted as $\alpha \& \beta$-thalassemia) [1]-[3]. At present, the fundamental test for thalassemia in large population is hematological screening and hemoglobin analysis [1]-[5]. First, use the mean corpuscular hemoglobin (MCH) and mean corpuscular volume (MCV) to determine whether it is the thalassaemias featuring microcytic hypochromic anemia. Following this, hemoglobin analysis is further performed to classify the thalassemia categories on the basis of the parameters of hemoglobin $\mathrm{F}$ and hemoglobin $\mathrm{A}_{2}$. Among them, $\mathrm{MCH}$ and $\mathrm{MCV}$ are most fundamental preliminary screening parameters with diagnostic value. In the conventional method, the cut-off values corresponding to $\mathrm{MCH}$ and $\mathrm{MCV}$ are $27.0 \mathrm{pg}$ and $80.0 \mathrm{fL}$, respectively [1]-[3]. Phenotype-positive subjects for thalassemia are those with $\mathrm{MCH} \leq 27.0 \mathrm{pg}$ or $\mathrm{MCV} \leq 80.0 \mathrm{fL}$. Individuals with low $\mathrm{MCH}$ or $\mathrm{MCV}(\mathrm{MCH} \leq 27.0 \mathrm{pg}$ or $\mathrm{MCV} \leq 80.0 \mathrm{fL})$ are usually further assessed using hemoglobin and DNA analyses for identifying the type of defect. In clinical application, MCH and MCV are usually used as simultaneous screening parameters. There are also some work try using MCV or MCH independently as screening parameter [6]-[8]. Therefore, it is significance to discuss the correlation between the hematological screening parameters $\mathrm{MCH}$ and $\mathrm{MCV}$ in large population for thalassemia. However, the work in this area has not been reported until date.

In the present study, a lot of blood samples of reproductive age persons were collected, and they were conducted for gene phenotype diagnosis of thalassemia and detected for hematological parameters MCH and MCV. According to the results of thalassemia phenotype diagnosis and hematology testing, the correlation analysis between MCH and MCV was further performed for each sample group of thalassemia and non-thalassemia carriers, which could be provide suitable strategy to select parameters and model for thalassemia screening in large population.

\section{Materials and Methods}

\subsection{Objects}

A total of 4920 peripheral blood samples $(2 \mathrm{~mL})$ of reproductive age persons were collected from Guangdong province of China, in which male and female were 2461, 2459 samples, respectively, their age ranged from 18 to 45. First, the hematological parameters MCH and MCV values of samples were measured, and then DNA analyses for thalassemia were conducted for the sample with $\mathrm{MCH}<27 \mathrm{pg}$ or MCV $<80 \mathrm{fL}$.

\subsection{Hematological Screening}

Hematological parameters MCH and MCV values of samples were measured using an XS-500i automatic blood cell analyzer (Sysmex Corporation Co., Ltd., Japan). The used reagents were mainly hemoglobin detection reagents, dilution liquid, and hemolysin, etc. (Guangzhou Huaxin Technology Co., Ltd., China).

\subsection{DNA Analyses for Thalassemia}

The gap point-polymerase chain reaction (Gap-PCR) method was used for gene diagnosis of $\alpha$-thalassemia, the 
used instruments were an S1000 Thermal circlers (Bio-rad Co., Ltd., USA), POWER 300 Gel electrophoresis system (Bio-rad Co., Ltd., USA), and Gel Doc ${ }^{\mathrm{TM}} 2000$ Gel Imaging System (Bio-rad Co., Ltd., USA), and 5415D High-speed centrifuge (Eppendorf Co., Ltd., Germany). The polymerase chain reaction-reverse dot blot hybridization (PCR-RDB) method was used for the gene diagnosis of $\beta$-thalassemia, the used instruments were S1000 Thermal circlers (BIO-RAD Co., Ltd., America), Combi-D24 Bunk Hybridization Ovens (Finemould Co., Ltd., Korea), and 5415D High-speed centrifuge (Eppendorf Co., Ltd., Germany). The used reagents were supplied by Shenzhen Yaneng Biotechnology Co., Ltd., China.

\subsection{Statistical Methods}

A total of 4920 peripheral blood samples $(2 \mathrm{~mL})$ of reproductive age persons were collected from Guangdong province of China, in which male and female were 2461, 2459 samples, respectively, their age ranged from 18 to 45. First, the hematological parameters MCH and MCV values of samples were measured, and then DNA analyses for thalassemia were conducted for the sample with $\mathrm{MCH}<27 \mathrm{pg}$ or MCV $<80 \mathrm{fL}$. Data analysis was performed with Microsoft Office Excel 2003 software. The correlation coefficient $(R)$ of measured MCH and MCV values for each sample group was calculated according to the following equation:

$$
R=\frac{\sum_{i=1}^{n}\left(\mathrm{MCH}_{i}-\mathrm{MCH}_{\mathrm{Ave}}\right)\left(\mathrm{MCV}_{i}-\mathrm{MCV}_{\mathrm{Ave}}\right)}{\sqrt{\sum_{i=1}^{n}\left(\mathrm{MCH}_{i}-\mathrm{MCH}_{\mathrm{Ave}}\right)^{2} \sum_{i=1}^{n}\left(\mathrm{MCV}_{i}-\mathrm{MCV}_{\mathrm{Ave}}\right)^{2}}}
$$

where $n$ is the number of samples in the sample group; $\mathrm{MCH}_{i}$ and $\mathrm{MCV}_{i}$ were the measured MCH and MCV values of $i^{\text {th }}$ sample, respectively; $\mathrm{MCH}_{\mathrm{Ave}}$ and $\mathrm{MCV}_{\mathrm{Ave}}$ were the mean measured values of $\mathrm{MCH}$ and MCV for the sample group, respectively. Numerical relation for MCH and MCV was fitted as the following linear equation:

$$
y=k x+b
$$

where $x$ represented MCH; $y$ represented MCV; $k$ and $b$ were the fitting coefficients.

\section{Results and Discussion}

Through gene diagnosis for thalassemia, all 4290 samples were composed by 4463 non-thalassemia and 457 thalassemia samples. Among 457 thalassemia samples, 311 were $\alpha$-thalassemia (included - -SEA $/ \alpha \alpha,-\alpha^{3.7} / \alpha \alpha$, $-\alpha^{4.2} / \alpha \alpha, \alpha \alpha^{\mathrm{QS}} / \alpha \alpha,--\mathrm{SEA} /-\alpha^{3.7},-\alpha^{3.7} / \alpha^{4.2}$, etc.), 133 were $\beta$-thalassemia (included $(41-42) / \beta$, (71 - 72)/ $\beta, 654 / \beta$, $\beta \mathrm{E} / \beta,-28 / \beta,-29 / \beta$, (IVSI-1) $/ \beta, 17 / \beta,(27-28) / \beta$, etc.), and 13 were $\alpha \& \beta$-thalassemia.

In accordance with non-thalassemia, thalassemia, $\alpha$-thalassemia, $\beta$-thalassemia, $\alpha \& \beta$-thalassemia and the entire group itself, a total of six sample groups were divided. Statistical analysis of the measured MCH and MCV values of six sample groups shown in Table 1.

The results of correlation analysis between the measured MCH and MCV values for six sample groups are summarized in Table 2. The relationship between the measured MCH and MCV values for six sample groups are shown in Figures 1-6.

The experimental results show that the correlation coefficient $R$ between the measured MCH and MCV values for the sample group of non-thalassemia was 0.880 , with a significant correlation; while those for the sample groups of thalassemia, $\alpha$-thalassemia, $\beta$-thalassemia, and $\alpha \& \beta$-thalassemia reached $0.968,0.966,0.962$, and 0.980 , respectively, with highly significant correlation; and that for the sample group of all samples also reached 0.965. It is interesting to observe that, the correlation coefficient between the measured MCH and MCV values for thalassemia carriers was significantly higher than those for non-thalassemia carriers.

\section{Conclusions}

Thalassemia has featuring microcytic hypochromic anaemia, the corresponding red blood cell count is relatively high, and the MCH and MCV values are significantly reduced. However, the correlation analysis of MCH and MCV for large population, especially the exact derivation of correlation coefficient and fitting regression equation based on different person groups has not been reported. 


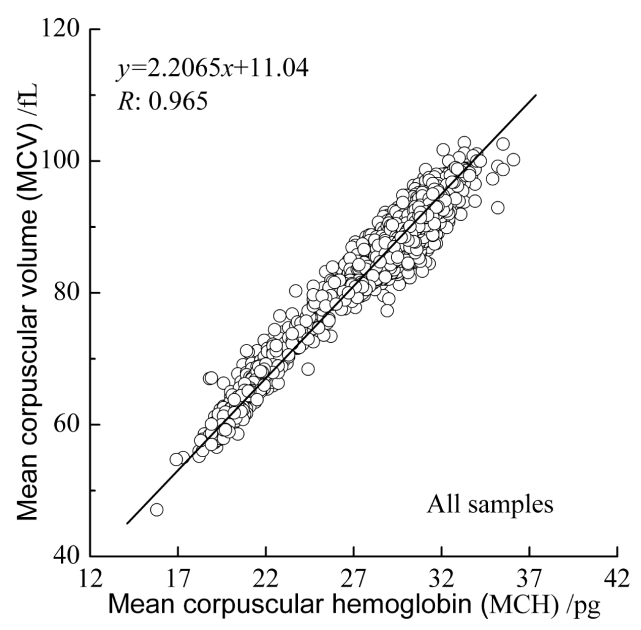

Figure 1. Relationship between the measured values of MCH and MCV for all samples.

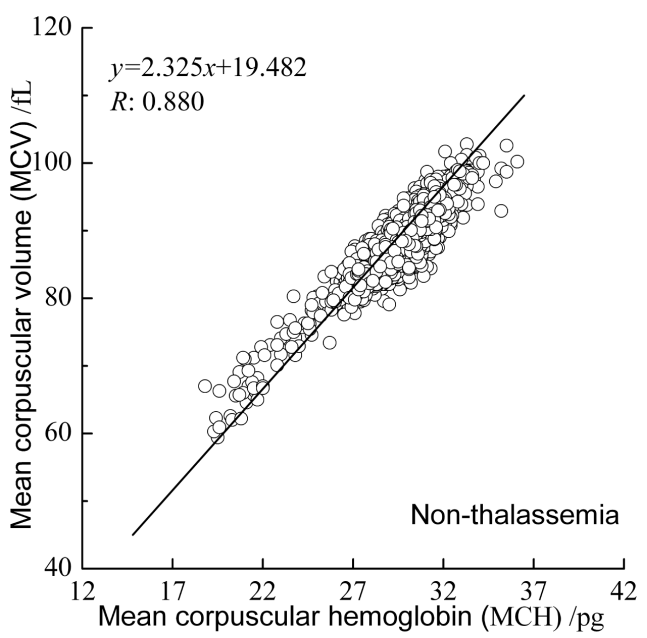

Figure 2. Relationship between the measured values of MCH and MCV for no-thalassemia carriers's samples.

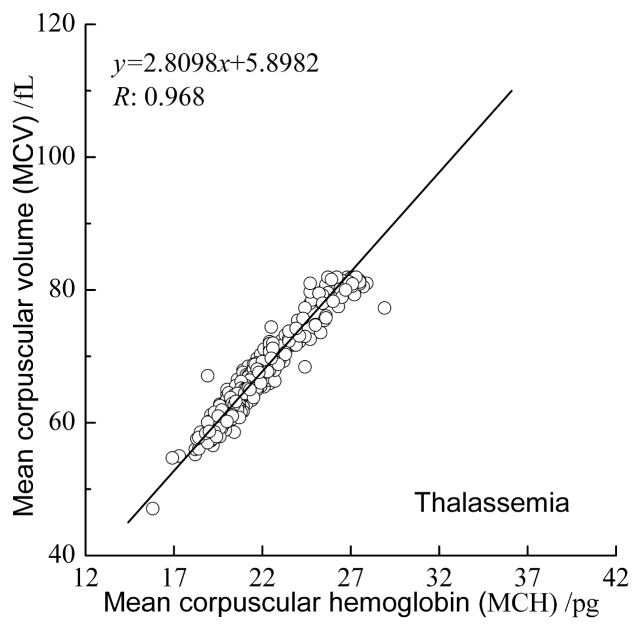

Figure 3. Relationship between the measured values of MCH and MCV for thalassemia carriers's samples. 


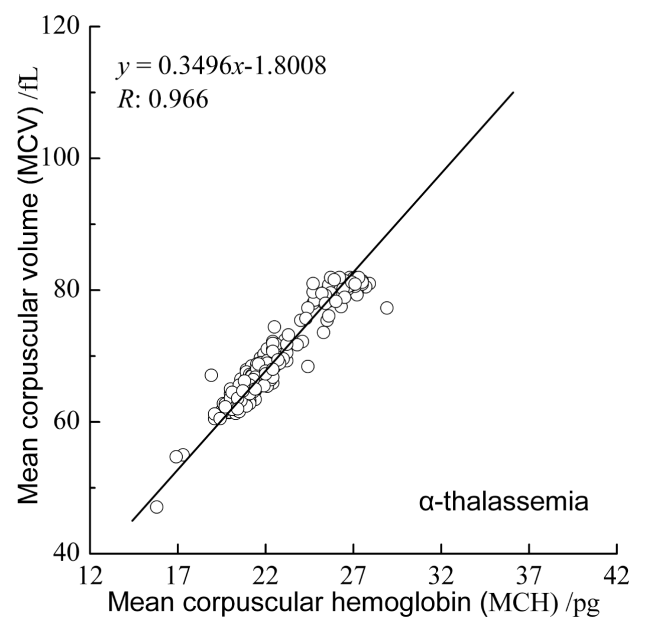

Figure 4. Relationship between the measured values of MCH and MCV for $\alpha$-thalassemia carriers's samples.

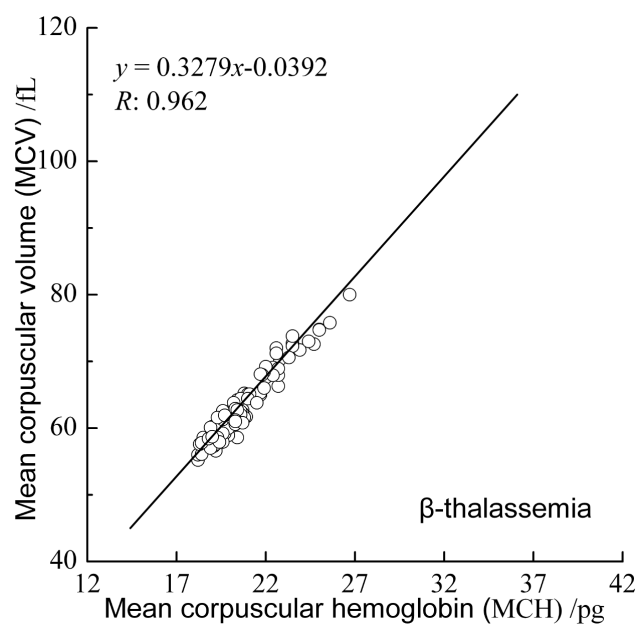

Figure 5. Relationship between the measured values of MCH and MCV for $\beta$-thalassemia carriers's samples.

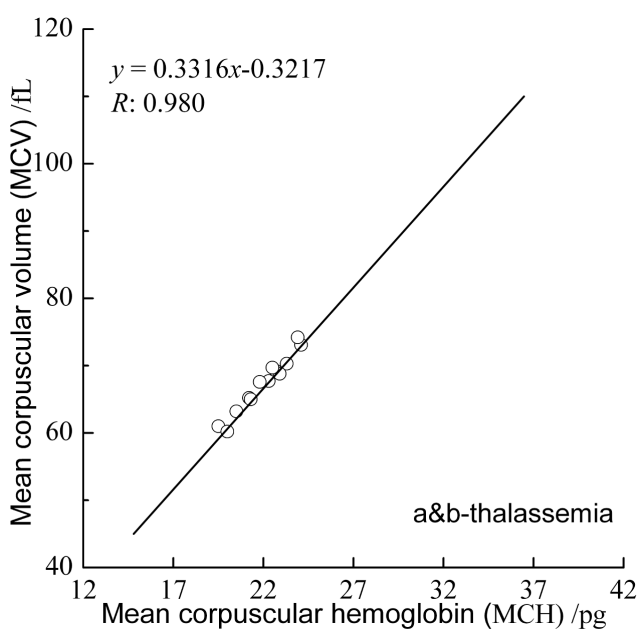

Figure 6. Relationship between the measured values of MCH and MCV for $\alpha \& \beta$-thalassemia carriers's samples. 
Table 1. Statistical analysis of the measured MCH and MCV values in each sample group.

\begin{tabular}{cccccccccc}
\hline \multirow{2}{*}{ Sample group } & Number & \multicolumn{4}{c}{ MCH (pg) } & \multicolumn{5}{c}{ MCV (fL) } \\
\cline { 3 - 10 } & & $\min$ & $\max$ & $\operatorname{mean}$ & SD & $\min$ & $\max$ & mean & SD \\
\hline All samples & 4920 & 15.8 & 36.1 & 28.99 & 2.83 & 47.1 & 102.8 & 86.61 & 7.63 \\
Non-thalassemia & 4463 & 18.8 & 36.1 & 29.73 & 1.56 & 59.4 & 102.8 & 88.60 & 4.12 \\
Thalassemia & 457 & 15.8 & 28.9 & 21.80 & 2.28 & 47.1 & 81.9 & 67.15 & 6.63 \\
$\alpha$-thalassemia & 311 & 15.8 & 28.9 & 22.37 & 2.31 & 47.1 & 81.9 & 69.13 & 6.39 \\
$\beta$-thalassemia & 133 & 18.2 & 26.7 & 20.45 & 1.63 & 55.2 & 80 & 62.50 & 4.79 \\
$\alpha$ \& $\beta$-thalassemia & 13 & 19.5 & 24.1 & 22.00 & 1.44 & 60.2 & 74.2 & 67.32 & 4.27 \\
\hline
\end{tabular}

Table 2. Correlation between the measured MCH and MCV values in each sample group.

\begin{tabular}{cccc}
\hline Sample group & Number & Correlation coefficient $(R)$ & Fitting equation \\
All samples & 4920 & 0.965 & $y=2.6065 x+11.04$ \\
Non-thalassemia & 4463 & 0.880 & $y=2.8098 x+5.8982$ \\
Thalassemia & 457 & 0.968 & $y=0.3496 x-1.8008$ \\
$\alpha$-thalassemia & 311 & 0.966 & $y=0.3279 x-0.0392$ \\
$\beta$-thalassemia & 133 & 0.962 & $y=0.3316 x-0.3217$ \\
$\alpha$ \& $\beta$-thalassemia & 13 & 0.980 &
\end{tabular}

Note: $x$ represented MCH and $y$ represented MCV in fitting equations.

In the present study, based on the thalassemia screening of large population (reproductive age) in Guangdong province of China, highly significant correlation between the measured MCH and MCV values for the thalassemia carriers were observed. The results indicated that the feasibility for thalassemia screening using MCV or $\mathrm{MCH}$ independently as parameter. The relevant results provided suitable strategy to select parameters and models for thalassemia screening in large population.

\section{Acknowledgements}

This work was supported by National Natural Science Foundation of China (No. 61078040), the Science and Technology Project of Guangdong Province of China (No. 2012B031800917), and the Science and Technology Project of Guangzhou of China (No. 2014Y2-00002).

\section{References}

[1] Galanello, R., Eleftheriou, A. and Traeger-Synodinos, J. (2003) Prevention of Thalassaemias and Other Haemoglobin Disorders. Vol. 1, Team up Creations Ltd., Nicosia.

[2] Xu, X.M., Zhou, Y.Q., Luo, G.X., et al. (2004) The Prevalence and Spectrum of Alpha and Beta Thalassaemia in Guangdong Province: Implications for the Future Health Burden and Population Screening. Journal of Clinical Pathology, 57, 517-522. http://dx.doi.org/10.1136/jcp.2003.014456

[3] Xiong, F., Sun, M., Zhang X., et al. (2010) Molecular Epidemiological Survey of Haemoglobinopathies in the Guangxi Zhuang Autonomous Region of Southern China. Clinical Genetics, 78, 139-148. http://dx.doi.org/10.1111/j.1399-0004.2010.01430.x

[4] George, E. and Ann, T.J. (2010) Genotype-Phenotype Diversity of Beta-Thalassemia in Malaysia: Treatment Options and Emerging Therapies. The Medical Journal of Malaysia, 65, 256-260.

[5] Cao, A., Rosatelli, M.C., Monni, G. and Galanello, R. (2002) Screening for Thalassemia: A Model of Success. Obstetrics and Gynecology Clinics of North America, 29, 305-328. http://dx.doi.org/10.1016/S0889-8545(01)00006-7

[6] He, Y.J., Yang, X.H., Ma, F.G. and Shu, J.C. (2005) The Value of the Combined Tests of Mean Corpuscular Volume, 
Red Cell Osmotic Fragility Test and Hemoglobin Electrophoresis for Diagnosis of Thalassemia. Chinese Journal of Laboratory Medicine, 28, 244-246.

[7] Zhang, X.H., Zhou, Y.J., Li, P.P., et al. (2006) Study on the Screening Program of Thalassemia and the Genotype and Hematologic Parameter among People of Productive Age in a Village, Nanning Guangxi. Chinese Journal of Epidemiology, 27, 769-772.

[8] Li, L.Y., Li, Q., Song, L.L., et al. (2012) The Value of MCV, MCH and $\mathrm{HbA}_{2}$ in Laboratory Screening of Thalassemia. Chinese Journal of Obstetrics and Gynecology, 47, 96-100. 
Scientific Research Publishing (SCIRP) is one of the largest Open Access journal publishers. It is currently publishing more than 200 open access, online, peer-reviewed journals covering a wide range of academic disciplines. SCIRP serves the worldwide academic communities and contributes to the progress and application of science with its publication.

Other selected journals from SCIRP are listed as below. Submit your manuscript to us via either submit@scirp.org or Online Submission Portal.
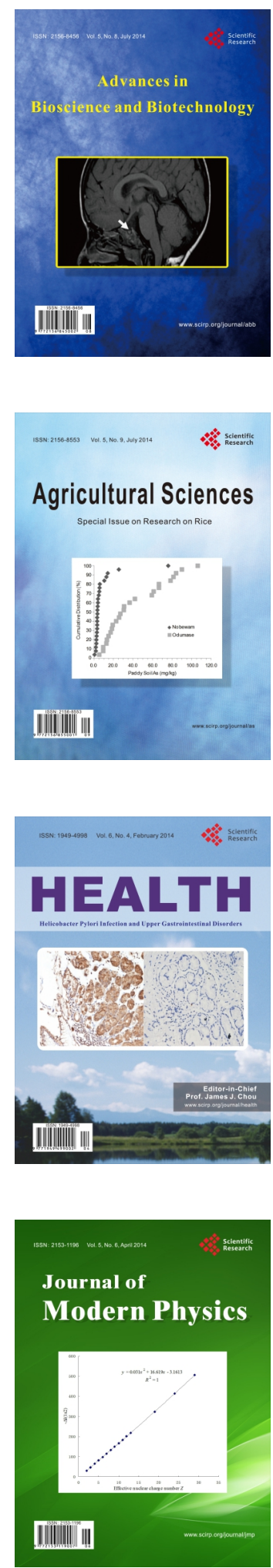
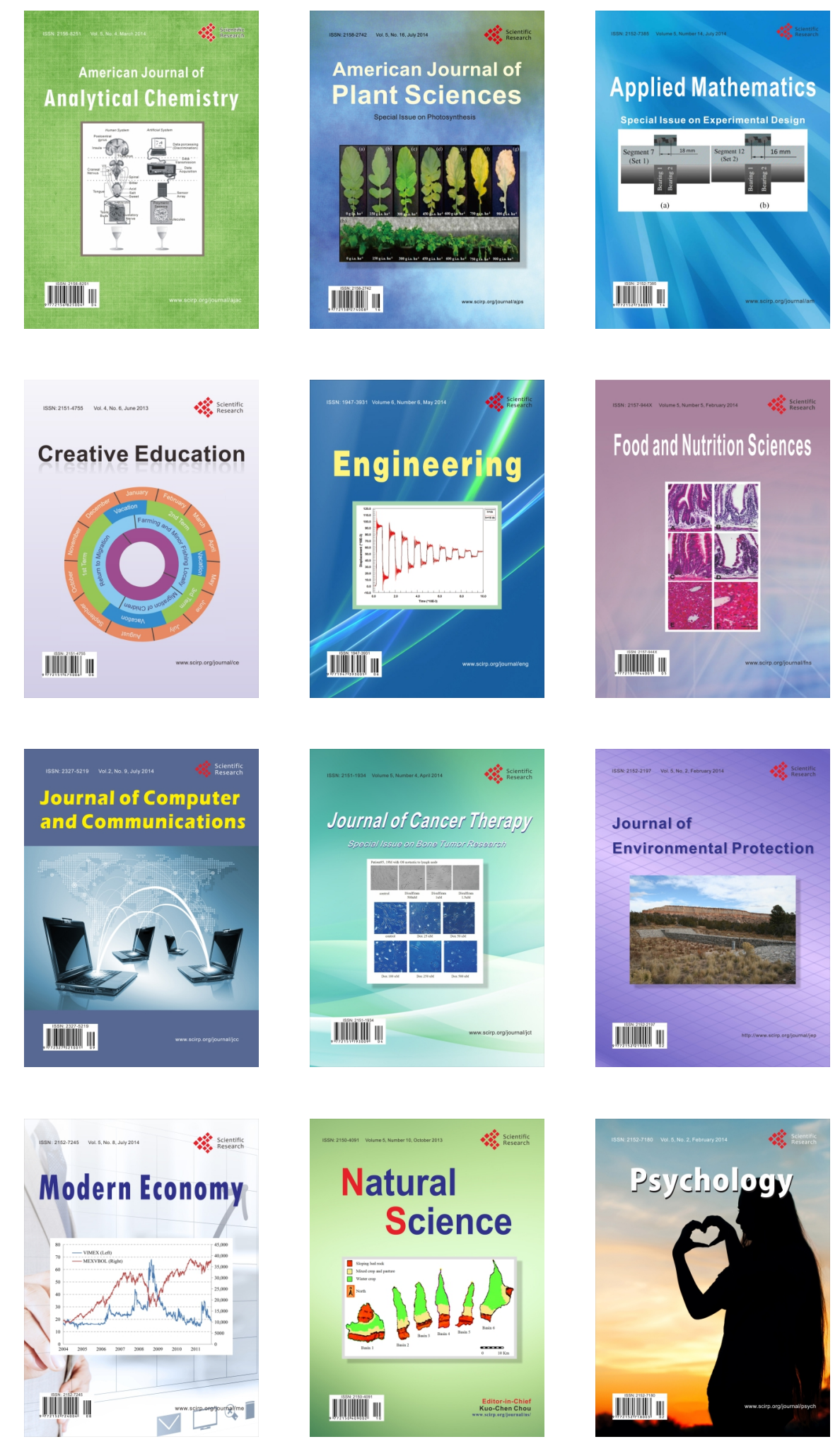